\title{
From pandemic Covid-19 to endemic Sars-2 Omicron Disease: is Long Covid still an issue?
}

\author{
Dr. Carolina Diamandis ${ }^{1}$, Olga Ivanova ${ }^{1}$, and Adrian Tudor Corresponding Author ${ }^{1}$
}

${ }^{1}$ Affiliation not available

January 31, 2022

\begin{abstract}
For many patients with severe Covid-19 disease of the pre-Omicron era, recovery is far from over with the end of acute symptoms. The authors of this paper have reviewed the currently available studies and conclude that Long-Covid appears to be a serious, sometimes even life-threatening syndrome. However, it seems to have good prospects for full recovery with multidisciplinary and evidence-based post-treatment care, which is, however, still lacking in Europe and North America. The Omicron variant appears to be a game changer in this regard as well. So far it seems that Long Covid is no common consequence of Sars-2-Omicron Disease.
\end{abstract}

\section{Hosted file}

Omicron and Long Covid.pdf available at https://authorea.com/users/410930/articles/554567from-pandemic-covid-19-to-endemic-sars-2-omicron-disease-is-long-covid-still-an-issue 